\title{
Obesity as a mortality risk factor in the medical ward: a case control study
}

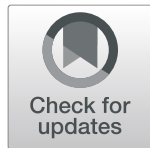

\author{
Shelly Soffer ${ }^{1,2^{*}}$ (D), Eyal Zimlichman ${ }^{3,4,5}$, Benjamin S. Glicksberg ${ }^{6,7}$, Orly Efros ${ }^{8,4}$, Matthew A. Levin ${ }^{9,10}$, \\ Robert Freeman ${ }^{9}$, David L. Reich ${ }^{10}$ and Eyal Klang ${ }^{4,5,11}$
}

\begin{abstract}
Background: Research regarding the association between severe obesity and in-hospital mortality is inconsistent. We evaluated the impact of body mass index (BMI) levels on mortality in the medical wards. The analysis was performed separately before and during the COVID-19 pandemic.

Methods: We retrospectively retrieved data of adult patients admitted to the medical wards at the Mount Sinai Health System in New York City. The study was conducted between January 1, 2011, to March 23, 2021. Patients were divided into two sub-cohorts: pre-COVID-19 and during-COVID-19. Patients were then clustered into groups based on BMI ranges. A multivariate logistic regression analysis compared the mortality rate among the BMl groups, before and during the pandemic.

Results: Overall, 179,288 patients were admitted to the medical wards and had a recorded BMI measurement. 149,098 were admitted before the COVID-19 pandemic and 30,190 during the pandemic. Pre-pandemic, multivariate analysis showed a "J curve" between BMI and mortality. Severe obesity (BMI > 40) had an aOR of 0.8 (95\% Cl:0.7-1.0, $p=0.018$ ) compared to the normal BMI group. In contrast, during the pandemic, the analysis showed a "U curve" between BMI and mortality. Severe obesity had an aOR of 1.7 (95\% Cl:1.3-2.4, $p<0.001$ ) compared to the normal BMI group.

Conclusions: Medical ward patients with severe obesity have a lower risk for mortality compared to patients with normal BMI. However, this does not apply during COVID-19, where obesity was a leading risk factor for mortality in the medical wards. It is important for the internal medicine physician to understand the intricacies of the association between obesity and medical ward mortality.
\end{abstract}

Keywords: Obesity, Hospital mortality, COVID-19, SARS-CoV-2

\section{Background}

Obesity has reached epidemic proportions worldwide. The prevalence of obesity is up to $40 \%$ among adults in the United States [1]. It is well recognized that individuals with obesity are more likely to suffer from diabetes, cardiovascular disease, and certain malignancies [2].

\footnotetext{
* Correspondence: soffer.shelly@gmail.com

'Internal Medicine B, Assuta Medical Center, Ashdod, Israel

${ }^{2}$ Ben-Gurion University of the Negev, Be'er Sheva, Israel

Full list of author information is available at the end of the article
}

Individuals with obesity require more hospital admissions compared with the non-obese population [3].

There is a strong association between body weight and mortality in the general population [4]. However, controversy exists regarding the effect of severe obesity on in-hospital mortality. Some studies have shown that obesity reduces overall inpatient mortality risk, a phenomenon termed as the "obesity paradox" [5-7], while others demonstrated an increased inpatient mortality risk $[8,9]$. However, these studies included a small

C C The Author(s). 2021 Open Access This article is licensed under a Creative Commons Attribution 4.0 International License, which permits use, sharing, adaptation, distribution and reproduction in any medium or format, as long as you give appropriate credit to the original author(s) and the source, provide a link to the Creative Commons licence, and indicate if changes were made. The images or other third party material in this article are included in the article's Creative Commons licence, unless indicated otherwise in a credit line to the material. If material is not included in the article's Creative Commons licence and your intended use is not permitted by statutory regulation or exceeds the permitted use, you will need to obtain permission directly from the copyright holder. To view a copy of this licence, visit http://creativecommons.org/licenses/by/4.0/ The Creative Commons Public Domain Dedication waiver (http://creativecommons.org/publicdomain/zero/1.0/) applies to the data made available in this article, unless otherwise stated in a credit line to the data. 
number of patients with severe obesity and focused on specific illnesses.

On March 11th, the World Health Organization declared the COVID-19 outbreak a pandemic. Shortly after, several risk factors for disease severity and mortality were determined $[10,11]$. Severe obesity was shown to be a significant independent risk factor for in-hospital mortality from COVID-19 [12-14].

In this study, we evaluated the impact of BMI levels on mortality in the medical wards. The analysis was performed separately before and during the COVID-19 pandemic.

\section{Methods}

\section{Study setting and data source}

An Institutional Review Board (IRB) approval was granted for this retrospective cohort study. The IRB committee waived informed consent.

We identified consecutive admissions to five hospitals in the Mount Sinai Health System, NY, USA (Mount Sinai Hospital, Mount Sinai Brooklyn, Mount Sinai Queens, Mount Sinai Morningside, and Mount Sinai West). Electronic health record (EHR) data were extracted from EPIC (Epic Systems Corporation, Verona WI). The study time frame was from January 1, 2011, to March 23, 2021.

\section{Study design Population}

We included patients admitted to the medical wards with a measurement of Body Mass Index (BMI). We excluded patients younger than 18 years old. We also excluded patients admitted to the intensive care unit (ICU).

The date March 1, 2020 was used to differentiate between two sub-cohorts: pre-COVID-19 pandemic and during-COVID-19 pandemic. This date was selected according to the time when COVID-19 pandemic started to rise, and the number of hospitalizations rapidly increased [15]. Patients that were hospitalized before March 1st but remained in the hospital after this date were included in the pre-COVID cohort.

\section{Variables}

The primary outcome was the association between BMI levels and all-cause in-hospital mortality. Covariates of interest included: age, sex, race, and comorbidities. Comorbidities were coded using the International Classification of Diseases, 10th Revision, Clinical Modification (ICD-10-CM), and grouped using the Healthcare Cost and Utilization Project diagnostic clinical classification software (CCS) [17]. They included: coronary artery disease (CAD), congestive heart failure (CHF), diabetes mellitus (DM), hypertension (HTN), chronic kidney disease (CKD), chronic obstructive pulmonary disease (COPD), and cancer. ICD-10 coded primary diagnoses at discharge were extracted from the EHR. Data regarding race was incomplete and in $31 \%$ of the patients this variable was categorized as "other". Data relating ethnicity was mostly missing and thus was not included in the analysis.

Data regarding SARS-CoV-2 nasopharyngeal swab polymerase chain reaction (PCR) tests were retrieved.

\section{BMI groups selection}

Recorded BMI measurements were retrieved from the EHR. Patients were clustered into groups based on BMI measurements: $<18.5 \mathrm{~kg} / \mathrm{m}^{2}, \quad 18.5-24.9 \mathrm{~kg} / \mathrm{m}^{2}, \quad 25-29.9$ $\mathrm{kg} / \mathrm{m}^{2}, 30-34.9 \mathrm{~kg} / \mathrm{m}^{2}, 35-39.9 \mathrm{~kg} / \mathrm{m}^{2}, \geq 40 \mathrm{~kg} / \mathrm{m}^{2}$ [16]. Severe obesity was defined as a BMI above $40 \mathrm{~kg} / \mathrm{m}^{2}$ [16].

\section{Statistical analysis}

Descriptive statistics were reported for all patient characteristics using means and standard deviation or medians with inter quartile range (IQR) for continuous variables and counts with percentages for categorical variables. Continuous variables were compared using either the unpaired t-test for two variables or 1-way analysis of variance (ANOVA) for more than two variables. Categorical variables were compared using the $\mathrm{x}^{2}$ test. Separate analyses were performed for the pre-COVID-19 pandemic and the during-COVID-19 pandemic cohorts.

Age distribution curves were plotted for different BMI groups. Gaussian kernel density estimates were fitted to the plots.

We employed the mutual information (MI) formula to identify primary diagnoses associated with severe obesity. Mutual information measures the statistical dependence between the severe obesity group (G) and a given diagnosis (d), as follows:

$$
\begin{aligned}
& \text { Mutual Information }=\sum \sum P(G, d) * \log \frac{P(G, d)}{P(G) P(d)} \\
& \begin{aligned}
P(G, d) & =\text { Joint probability of } G \text { and } d ; P(G) \\
& =\text { probability of } G ; P(d)=\text { probability of } d .
\end{aligned}
\end{aligned}
$$

In addition to MI, we also determined odds ratios (OR). Multivariate logistic regression models compared rates of mortality between the different BMI groups. Separate models were built for the pre-COVID-19 pandemic and during-COVID-19 pandemic cohorts. The models were adjusted for demographics (age, sex, race) and comorbidities (CAD, CHF, DM, HTN, CKD, COPD, cancer). Patients with normal BMI measurements (18.5-24.9 kg/ $\mathrm{m}^{2}$ ) were used as reference. Correlation matrices were constructed to assess the possible collinearity between covariates. All covariate correlations were below 0.46 
(Appendix Fig. 1A, B). Adjusted odds ratios (aOR) with 95\% confidence intervals $(\mathrm{CI})$ were reported.

C-statistic was calculated for the multivariate models. Bootstrapping validations (1000 bootstrap resamples) were used to calculate $95 \%$ confidence intervals (CI) for all metrics.

All analyses were conducted with Python (Python software foundation, Version 3.6.5).

\section{Results}

The cohort included 179,288 patients that were admitted to the medical wards. Of those, 149,098 were hospitalized before the COVID-19 pandemic and 30,190 during the pandemic. Figure 1 presents the study inclusion flow chart.

The median age of the entire cohort was 65 years (IQR 51-78), and 91,312 (50.9\%) patients were females (Table 1$)$. The overall in-hospital mortality rate was $2.3 \%(n=4200)$. The mortality rate was significantly higher during the COVID-19 pandemic (3.3\% versus $2.2 \%, P<0.001$ ).

Of the during-COVID-19 cohort, 4602 / 30,190 (15.2\%) had a positive in-hospital COVID-19 PCR test. The mortality rate was significantly higher among the PCR positive group: there were 527 patients who died out of 4602 with a positive PCR test (11.5\%) in comparison to 463 patients who died out of 25,588 with a nonpositive PCR test (1.8\%), $P<0.001$.

\section{BMI levels}

The BMI distribution was right-skewed (Appendix Fig. 2), with a median of $26.1 \mathrm{~kg} / \mathrm{m}^{2}$ (IQR 22.4-26.1). Obesity
$\left(\mathrm{BMI}{ }^{3} 30 \mathrm{~kg} / \mathrm{m}^{2}\right.$ ) was found in $28.5 \%$ in the pre-COVID19 and $29.2 \%$ in the COVID-19 cohort. Severe obesity $\left(\mathrm{BMI}^{3} 40 \mathrm{~kg} / \mathrm{m}^{2}\right)$ was found in $6.2 \%$ of the pre-COVID-19 and $6.0 \%$ in the COVID-19 pandemic cohort.

In both cohorts, patients' median age decreased with higher BMI levels (Fig. 2A, B). Patients with severe obesity had the lowest median age (58 years in the preCOVID-19 and 59 years in the COVID-19 pandemic cohort) (Appendix Tables 1 and 2). Underweight patients had the highest median age (70 years in the pre-COVID19 and 71 years in the COVID-19 cohort). Patients with severe obesity were more likely to have comorbidities such as DM, HTN, COPD, and CHF and were less likely to have malignancy.

Appendix Table 3A, B demonstrate the ten most common primary diagnoses in the severe obesity groups. In the pre-COVID-19 cohort, cellulitis had the greatest association with severe obesity $(7.5 \%$ vs. $2.9 \%$, OR of $2.8, P<$ $0.001)$, followed by shortness of breath $(7.2 \%$ vs. $3.3 \%$, OR of 2.3, $\mathrm{P}<0.001)$. Among the during-COVID-19 cohort, shortness of breath had the greatest association with severe obesity $(7.4 \%$ vs. $3.1 \%$, OR of $2.5, \mathrm{P}<0.001)$, followed by cellulitis ( $5.8 \%$ vs. $2.2 \%$, OR of $2.8, p<0.001)$.

\section{Multivariable models}

Crude mortality rates, stratified by BMI levels, are presented in Fig. 3. Mortality rates were higher during the COVID-19 pandemic. Subgroup analysis by age and sex is presented in Appendix Fig. 3A-D.

The multivariable models had C-statistic of 0.71 (95\% CI: $0.71-0.72)$ in the pre-COVID-19 pandemic cohort,

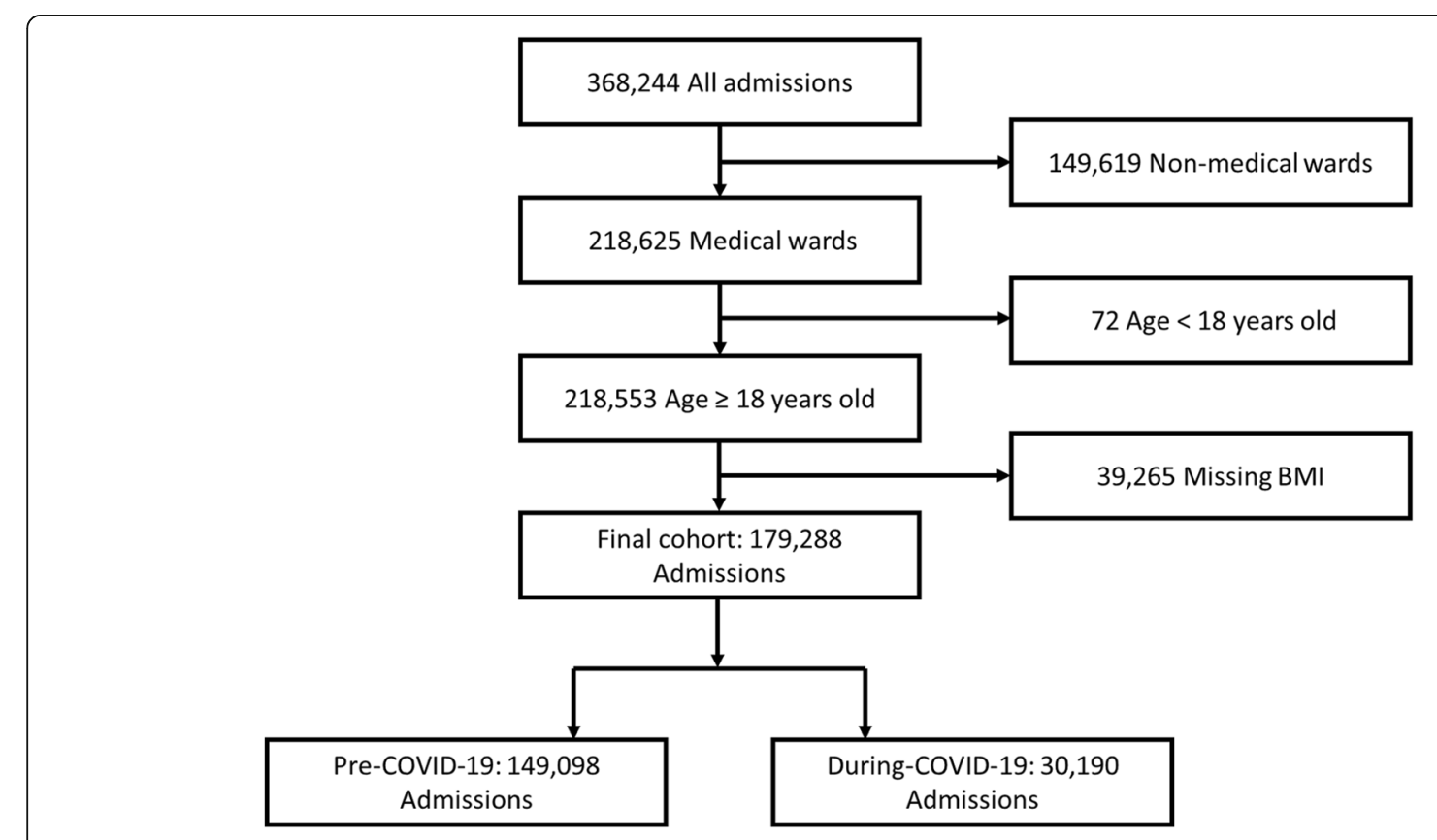

Fig. 1 Study flow chart 
Table 1 Clinical and demographic characteristics of patient cohort before and during the COVID-19 pandemic $(n=179,288)$

\begin{tabular}{|c|c|c|c|c|}
\hline & $\begin{array}{l}\text { Entire Cohort } \\
(n=179,288)\end{array}$ & $\begin{array}{l}\text { pre-COVID-19 } \\
(n=149,098,83.2 \%)\end{array}$ & $\begin{array}{l}\text { during-COVID-19 } \\
(n=30,190,16.8 \%)\end{array}$ & $P$ value \\
\hline \multicolumn{5}{|l|}{ BMl groups } \\
\hline$<18.5$ kg/m², N. (\%) & $10,775(6.0)$ & $8950(6.0)$ & $1825(6.0)$ & $<0.001$ \\
\hline $18.5-24.9 \mathrm{~kg} / \mathrm{m}^{2}, \mathrm{~N} .(\%)$ & $64,757(36.1)$ & $54,225(36.4)$ & $10,532(34.9)$ & $<0.001$ \\
\hline $25-29.9$ kg/m², N. (\%) & $52,436(29.2)$ & $43,426(29.1)$ & $9010(29.8)$ & $<0.001$ \\
\hline $30-34.9$ kg/m², N. (\%) & $27,576(15.4)$ & $22,813(15.3)$ & $4763(15.8)$ & $<0.001$ \\
\hline $35-39.9$ kg/m², N. (\%) & $12,742(7.1)$ & $10,500(7.0)$ & $2242(7.4)$ & $<0.001$ \\
\hline$\geq 40 \mathrm{~kg} / \mathrm{m}^{2}, \mathrm{~N} .(\%)$ & $11,002(6.1)$ & $9184(6.2)$ & $1818(6.0)$ & $<0.001$ \\
\hline \multicolumn{5}{|l|}{ Demographics } \\
\hline Age, median (IQR), y & $65.0(51.0-78.0)$ & $65.0(51.0-78.0)$ & $66.0(53.0-78.0)$ & $<0.001$ \\
\hline Female, N. (\%) & $91,308(50.9)$ & $76,785(51.5)$ & $14,523(48.1)$ & $<0.001$ \\
\hline Black, N. (\%) & $48,515(27.1)$ & $39,362(26.4)$ & $9153(30.3)$ & $<0.001$ \\
\hline White, N. (\%) & $57,700(32.2)$ & $48,241(32.4)$ & $9459(31.3)$ & $<0.001$ \\
\hline \multicolumn{5}{|l|}{ Comorbidities } \\
\hline$C A D, N .(\%)$ & $44,505(24.8)$ & $35,740(24.0)$ & 8765 (29.0) & $<0.001$ \\
\hline CHF, N. (\%) & $40,546(22.6)$ & $32,995(22.1)$ & $7551(25.0)$ & $<0.001$ \\
\hline DM, N. (\%) & $66,022(36.8)$ & $53,796(36.1)$ & $12,226(40.5)$ & $<0.001$ \\
\hline HTN, N. (\%) & $92,294(51.5)$ & $74,896(50.2)$ & $17,398(57.6)$ & $<0.001$ \\
\hline CKD, N. (\%) & $33,031(18.4)$ & $27,150(18.2)$ & $5881(19.5)$ & $<0.001$ \\
\hline COPD, N. (\%) & $24,788(13.8)$ & $20,613(13.8)$ & 4175 (13.8) & 0.99 \\
\hline Cancer, N. (\%) & $51,659(28.8)$ & $42,591(28.6)$ & $9068(30.0)$ & $<0.001$ \\
\hline Past or present smoking, N. (\%) & $75,186(41.9)$ & $63,967(42.9)$ & $11,219(37.2)$ & $<0.001$ \\
\hline \multicolumn{5}{|l|}{ Mortality } \\
\hline Mortality rate, N. (\%) & $4200(2.3)$ & $3210(2.2)$ & 990 (3.3) & $<0.001$ \\
\hline
\end{tabular}

Abbreviations: BMI - body mass index; IQR - interquartile range; CAD - Coronary artery disease; CHF - Congestive heart failure; DM - Diabetes mellitus; HTN - hypertension; CKD - Chronic kidney disease; COPD - Chronic obstructive pulmonary disease
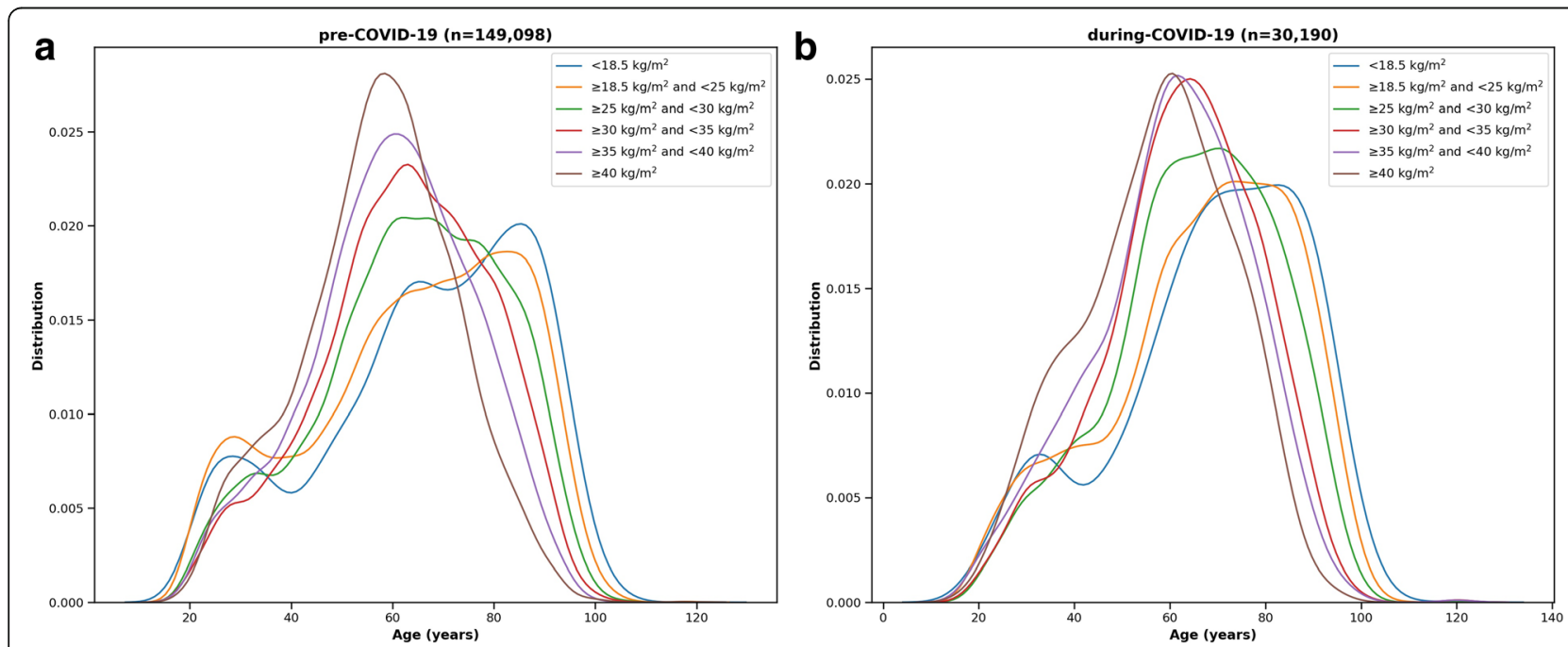

Fig. 2 Histograms demonstrating age distributions according to different BMl groups for the $\mathbf{A}$ pre-COVID-19 cohort and $\mathbf{B}$ during-COVID-19 cohort 


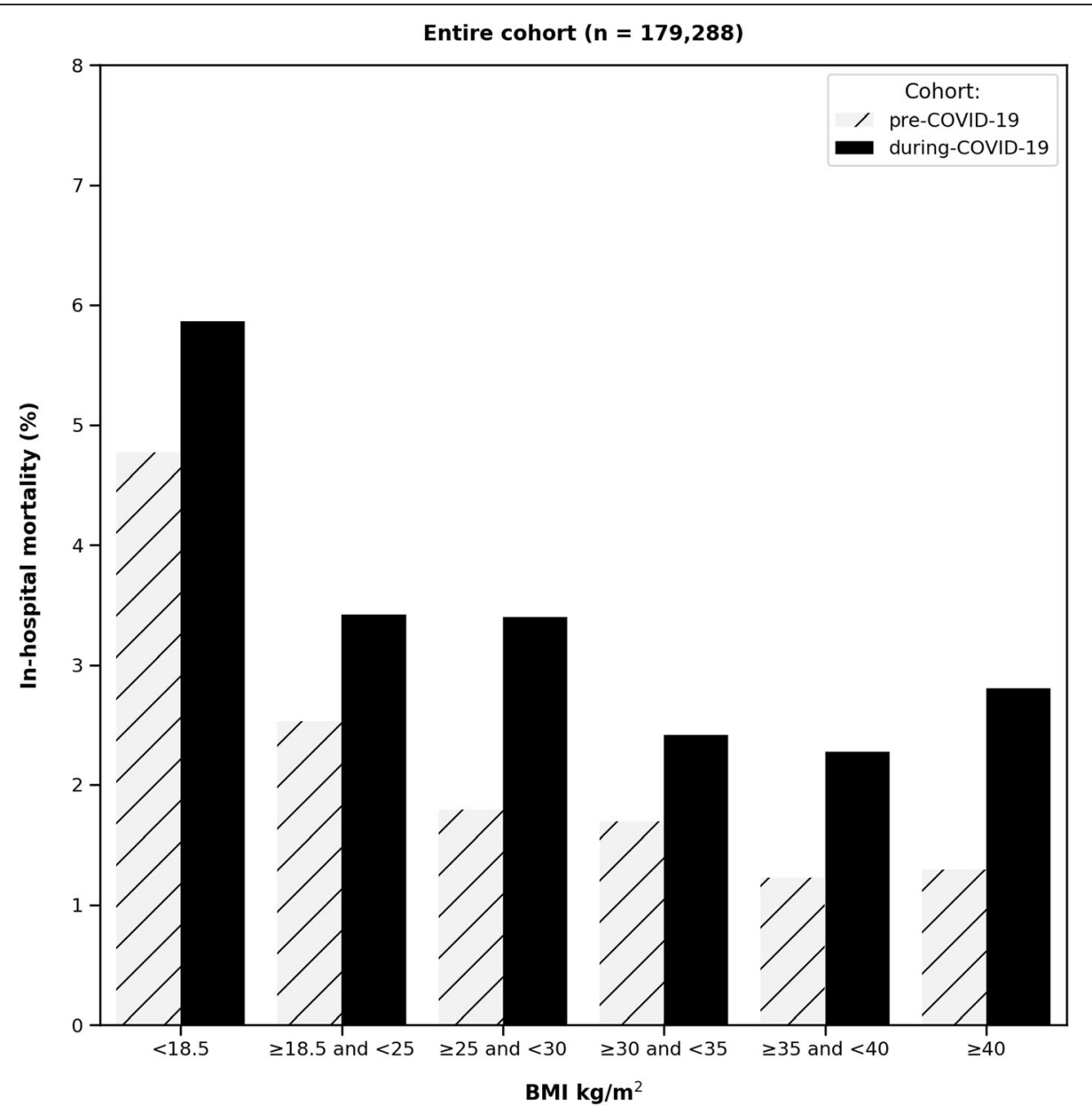

Fig. 3 In-hospital mortality rates according to BMl for the pre-COVID-19 cohort and the during-COVID-19 cohort

and C-statistic of 0.75 (95\% CI: 0.73-0.76) in the duringCOVID-19 pandemic cohort.

In the pre-COVID-19 cohort, multivariable analysis showed a mild "J curve" (Fig. 4A). Severe obesity had an aOR of 0.8 (95\% CI 0.7-1.0, $p=0.018$ ) compared to normal BMI. Underweight patients had a significantly higher mortality rate in this cohort compared to the other BMI groups, with an aOR of 1.8 (95\% CI: 1.6-2.0, $p<0.001)$.

In contrast, in the during-COVID-19 cohort, multivariable analysis showed a "U curve" (Fig. 4B). Severe obesity had an aOR of 1.7 (95\% CI 1.3-2.4, p < 0.001) compared to normal BMI. Underweight patients had the secondhighest mortality rate compared to the other BMI groups, with an aOR of 1.6 (95\% CI 1.3-2.0, p < 0.001).

\section{Discussion}

This multi-site study evaluated the intricate relationship between BMI levels and mortality in the medical ward.

While obesity is an extremely common morbidity that affects patients in the medical wards, the literature regarding its effect on in-hospital mortality is inconsistent.
Previous studies showed obesity to be either associated with a decline, an unchanged, or an elevated risk of mortality. Nevertheless, most studies did not refer to the severe obesity group $\left(B M I \geq 40 \mathrm{~kg} / \mathrm{m}^{2}\right)$ [7, 17-21]. Studies that explicitly included severe obesity were based on a limited number of subjects [6, 22-24]. Additionally, they were limited to specific diagnoses such as acute myocardial infarction and intracerebral hemorrhage. In this study, we focused on the all-cause mortality in the medical wards. We included a large multi-site cohort of more than 10,000 patients with severe obesity. We found that before COVID-19, the risk for mortality was higher for underweight patients, lowest for the obese group, and marginally lower for the severely obese. In contrast, in the past year, since the COVID-19 emerged, patients with severe obesity had twice the adjusted risk for mortality. Indeed, during the pandemic, severe obesity was the highest mortality risk factor in the medical wards.

Several mechanisms were proposed to elucidate the "obesity paradox". It has been theorized that obesity is a marker of nutritional reserve that can be utilized in 

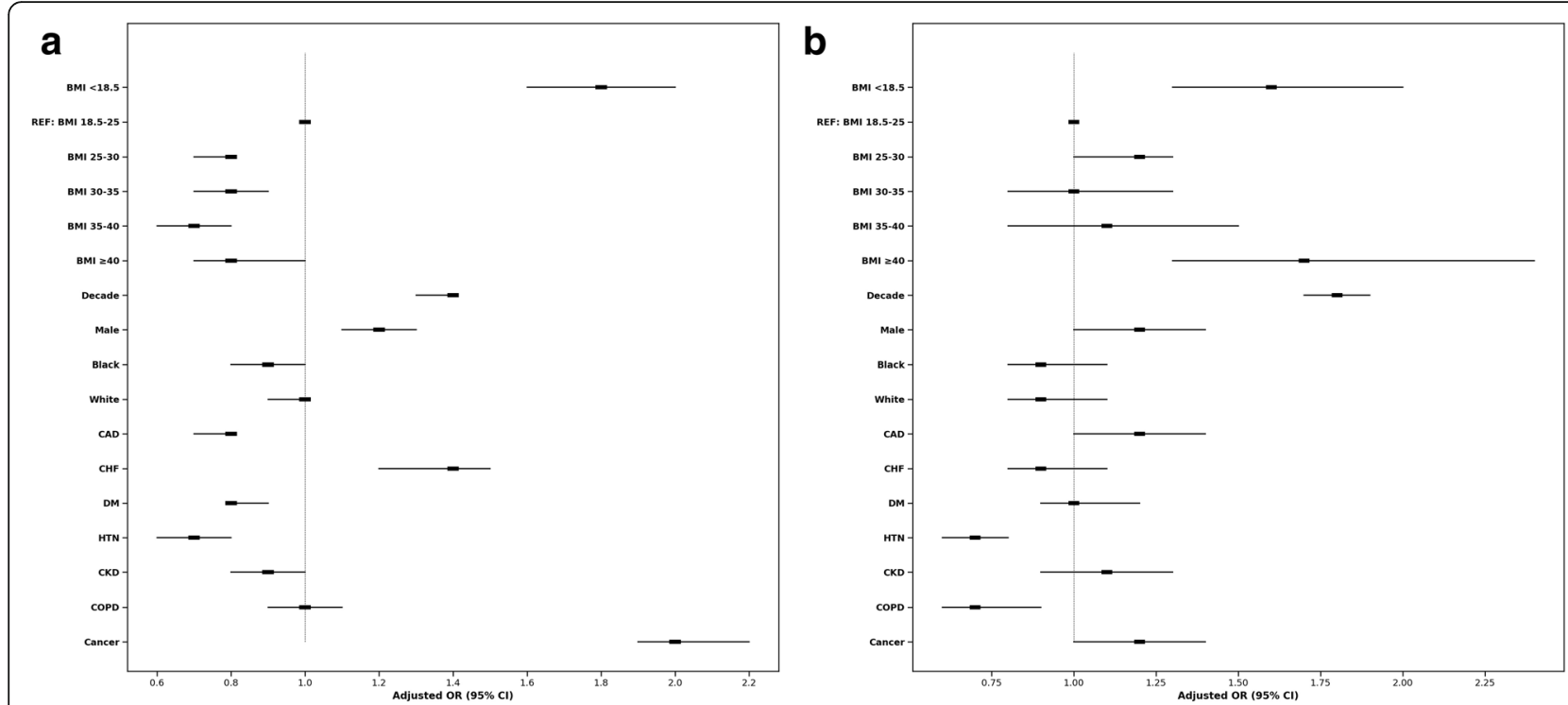

Fig. 4 Forest plots presenting multivariable analyses comparing rates of in-hospital mortality between BMI groups for the A pre-COVID-19 cohort and $\mathbf{B}$ during-COVID-19 cohort. The models were adjusted for age decile, male sex, race, CAD, CHF, HTN, DM, CKD, COPD, cancer. Patients with normal BMl measurements (18.5-24.9 kg/m²) were used as the reference group. Abbreviations: Coronary artery disease (CAD); Congestive heart failure (CHF); Hypertension (HTN); Diabetes mellitus (DM); Body mass index (BMI); Chronic kidney disease (CKD)

conditions with high metabolic demands. In contrast, end-stage diseases that inevitably have a high mortality rate and are frequently treated in the medical wards are characterized by cachexia and protein catabolism [25, 26]. In our study, although underweight patients had a lower risk for cardiovascular comorbidities, they were older and had a higher malignancy rate. Elderly individuals are more prone to unintentional weight loss and are at a higher risk for malnutrition [18].

During the COVID-19 pandemic, these factors may not play a similar role. Several mechanisms have been suggested to explain the unique link between obesity and COVID-19 severity [27-31]. The expanded mass of adipose tissue contributes to a prothrombotic state and chronic inflammation [32]. Abnormal cytokine production and microthrombi formation were proposed as key mediators of COVID-19 severity in patients with obesity. Obesity is characterized by physiological lung alteration that can result in ventilatory dysfunction [33]. The decreased functional residual capacity and hypoxemia observed in patients with obesity make them vulnerable to a more severe COVID-19 illness [34]. Additionally, the management of obese patients is further complicated by difficulties in several medical interventions such as intubation and prone positioning [35].

Internal medicine physicians play an important role in the treatment of patients with severe obesity. This clearly applies to the period before as well as during the COVID-19 pandemic. Physicians are educated according to the assumption that obesity is a risk factor for mortality. However, it is important to have a more indepth understanding of the context of this premise.

Our study has several limitations. This is a retrospective study and may suffer from inherent biases. The two cohorts demonstrated substantial differences in age, comorbidities, and demographics. Although a multivariate regression analysis was performed with adjustment to age and comorbidities, we could not incorporate all possible influencing factors. Therefore, a selective survival bias may remain. Moreover, data on disease severity at presentation was not available in this study. It is possible that physicians tended to hospitalize patients with obesity in a milder condition, influencing the cohort observed prognosis. Although we demonstrated an association between BMI and mortality, a cause-and-effect relationship cannot be deduced between these two variables. Additionally, even though this study was based on a multi-site cohort, it was limited to the New York population. Lastly, we used BMI to define severe obesity. Although indices that reflect adipose tissue distribution were also shown to correlate with COVID-19 severity, they were not available for this work.

\section{Conclusion}

Medical ward patients with severe obesity have a lower risk for mortality compared to patients with normal BMI. However, this does not apply during COVID-19, where obesity was a leading risk factor for mortality in the medical wards. It is important for the internal medicine physician to understand the intricacies of the association between obesity and medical ward mortality. 


\section{Abbreviations}

BMI: Body mass index; IRB: Institutional Review Board; EHR: Electronic health record; ICU: Intensive care unit; CCS: Clinical classification software; CAD: Coronary artery disease; CHF : Congestive heart failure; DM: Diabetes mellitus; HTN: Hypertension; CKD: Chronic kidney disease; COPD: Chronic obstructive pulmonary disease; PCR: Polymerase chain reaction; MI: Mutual information; OR: Odds ratios; Cl: Confidence intervals

\section{Supplementary Information}

The online version contains supplementary material available at https://doi. org/10.1186/s12902-021-00912-5.

\section{Additional file 1.}

\section{Acknowledgements}

Not applicable.

\section{Authors' contributions}

EK had full access to all of the data in the study and takes responsibility for the integrity of the data and the accuracy of the data analysis. SS, EK designed the study protocol, performed the data analysis. EK, MAL, RF collected the data. EK, SS drafted the manuscript. All authors offered intellectual input and provided critical revision of the manuscript. All authors read and approved the final version of the manuscript.

\section{Funding}

The authors have not declared a specific grant for this research from any funding agency in the public, commercial or not-for-profit sectors.

\section{Availability of data and materials}

The data used to support the findings of this study are available from the corresponding author upon reasonable request.

\section{Declarations}

\section{Ethics approval and consent to participate}

An Institutional Review Board (IRB) approval was granted for this retrospective cohort study. The IRB committee waived informed consent. The protocol was approved by the Ethics Committee of the Mount Sinai Health System.

\section{Consent for publication}

Not applicable.

\section{Competing interests}

Authors have no conflict of interests.

\section{Author details}

${ }^{1}$ Internal Medicine B, Assuta Medical Center, Ashdod, Israel. ${ }^{2}$ Ben-Gurion University of the Negev, Be'er Sheva, Israel. ${ }^{3}$ Hospital Management, Sheba Medical Center, Tel Hashomer, Israel. ${ }^{4}$ Sackler Medical School, Tel Aviv University, Tel Aviv, Israel. ${ }^{5}$ Sheba Talpiot Medical Leadership Program, Tel Hashomer, Israel. ${ }^{6}$ Hasso Plattner Institute for Digital Health at Mount Sinai, Icahn School of Medicine at Mount Sinai, New York, NY, USA. 'Department of Genetics and Genomic Sciences, Icahn School of Medicine at Mount Sinai, New York, NY, USA. ${ }^{8}$ Thrombosis \& Hemostasis Unit, Sheba Medical Center, Tel Hashomer, Israel. ${ }^{9}$ Institute for Healthcare Delivery Science, Department of Population Health Science and Policy, Icahn School of Medicine at Mount Sinai, New York, USA. ${ }^{10}$ Department of Anesthesiology, Perioperative and Pain Medicine, Icahn School of Medicine at Mount Sinai, New York, USA.

${ }^{11}$ Department of Diagnostic Imaging, Sheba Medical Center, Tel Hashomer, Israel.

Received: 6 June 2021 Accepted: 5 December 2021

Published online: 06 January 2022

\section{References}

1. Tsai AG, Bessesen DH: Obesity. Annals of Internal Medicine 2019, 170(5): Itc33-itc48.
2. Prevention NTFot. Obesity To: Overweight, obesity, and health risk. Arch Intern Med 2000, 160(7):898-904.

3. Chen Y, Jiang Y, Mao Y. Hospital admissions associated with body mass index in Canadian adults. Int J Obes. 2007;31(6):962-7.

4. $\mathrm{Xu} \mathrm{H}$, Cupples LA, Stokes A, Liu C-T. Association of obesity with mortality over 24 years of weight history: findings from the Framingham heart study. JAMA Netw Open. 2018;1(7):e184587-7

5. Schetz $M$, De Jong A, Deane AM, Druml W, Hemelaar P, Pelosi $P$, et al. Obesity in the critically ill: a narrative review. Intensive Care Med. 2019:1-13.

6. Persaud SR, Lieber AC, Donath E, Stingone JA, Dangayach NS, Zhang X, et al. Obesity paradox in intracerebral hemorrhage: national inpatient sample analysis. Stroke. 2019;50(4):999-1002.

7. Camprubi M, Cabrera S, Sans J, Vidal G, Salvadó T, Bardají A. Body mass index and hospital mortality in patients with acute coronary syndrome receiving care in a university hospital. J Obes. 2012;2012.

8. Ghanta RK, LaPar DJ, Zhang Q, Devarkonda V, Isbell JM, Yarboro LT, et al. Obesity increases risk-adjusted morbidity, mortality, and cost following cardiac surgery. J Am Heart Assoc. 2017;6(3):e003831.

9. Akinyemiju T, Sakhuja S, Vin-Raviv N. In-hospital mortality and post-surgical complications among cancer patients with metabolic syndrome. Obes Surg. 2018;28(3):683-92.

10. Richardson S, Hirsch JS, Narasimhan M, Crawford JM, McGinn T, Davidson KW, et al. Presenting characteristics, comorbidities, and outcomes among 5700 patients hospitalized with COVID-19 in the new York City area. Jama. 2020.

11. Chen R, Liang W, Jiang M, Guan W, Zhan C, Wang T, et al. Risk factors of fatal outcome in hospitalized subjects with coronavirus disease 2019 from a nationwide analysis in China. Chest. 2020

12. Klang E, Kassim G, Soffer S, Freeman R, Levin MA, Reich DL. Morbid obesity as an independent risk factor for COVID-19 mortality in hospitalized patients younger than 50. Obesity. 2020.

13. Tartof SY, Qian L, Hong V, Wei R, Nadjafi RF, Fischer H, et al. Obesity and mortality among patients diagnosed with COVID-19: results from an integrated health care organization. Ann Intern Med. 2020;173(10):773-81.

14. Kalligeros M, Shehadeh F, Mylona EK, Benitez G, Beckwith CG, Chan PA, Mylonakis E. Association of Obesity with disease severity among patients with COVID-19. 2020.

15. Delahoy MJ, Ujamaa D, Whitaker M, O'Halloran A, Anglin O, Burns E, Cummings C, Holstein R, Kambhampati AK, Milucky J. Hospitalizations associated with COVID-19 among children and adolescents-COVID-NET, 14 states, march 1, 2020-august 14, 2021. 2021.

16. Weir CB, Jan A. BMI classification percentile and cut off points. StatPearls [Internet] 2019.

17. Chatterjee K, Gupta T, Goyal A, Kolte D, Khera S, Shanbhag A, et al. Association of obesity with in-hospital mortality of cardiogenic shock complicating acute myocardial infarction. Am J Cardiol. 2017;119(10): 1548-54.

18. Landi F, Onder G, Gambassi G, Pedone C, Carbonin P, Bernabei R. Body mass index and mortality among hospitalized patients. Arch Intern Med. 2000;160(17):2641-4.

19. Cereda E, Klersy C, Hiesmayr M, Schindler K, Singer P, Laviano A, et al. Body mass index, age and in-hospital mortality: the NutritionDay multinational survey. Clin Nutr. 2017:36(3):839-47.

20. Akinyemiju T, Meng Q, Vin-Raviv N. Association between body mass index and in-hospital outcomes: analysis of the nationwide inpatient database. Medicine. 2016;95(28)

21. Buettner HJ, Mueller C, Gick M, Ferenc M, Allgeier J, Comberg T, et al. The impact of obesity on mortality in UA/non-ST-segment elevation myocardial infarction. Eur Heart J. 2007;28(14):1694-701.

22. Alexopoulos A-S, Fayfman M, Zhao L, Weaver J, Buehler L, Smiley D, et al. Impact of obesity on hospital complications and mortality in hospitalized patients with hyperglycemia and diabetes. BMJ Open Diabetes Res Care. 2016;4(1).

23. Harris $C$, Abougergi M, Wright S. Clinical outcomes among morbidly obese patients hospitalized with diabetic foot complications. Clin Obes. 2019;9(1): e12285

24. Tlayjeh H, Arabi YM, Ferguson ND, Zhou Q, Lamontagne F, Arroliga A, et al. Body mass index and mortality in subjects with ARDS: post-hoc analysis of the OSCILLATE trial. Respir Care. 2019;64(9):1042-8.

25. Song EK, Lee Y, Moser DK. The link of unintentional weight loss to cardiac event-free survival in patients with heart failure brief title: weight loss, 
depressive symptoms, and hsCRP. The Journal of Cardiovascular Nursing. 2014;29(5):439.

26. Bergström J, Wang T, Lindholm B. Factors contributing to catabolism in end-stage renal disease patients. Miner Electrolyte Metab. 1998;24(1):92-101.

27. Lockhart SM, O'Rahilly $S$. When two pandemics meet: why is obesity associated with increased COVID-19 mortality? Med 2020

28. Stefan N, Birkenfeld AL, Schulze MB. Global pandemics interconnected-obesity, impaired metabolic health and COVID-19. Nat Rev Endocrinol. 2021:1-15.

29. Rychter AM, Zawada A, Ratajczak AE, Dobrowolska A, Krela-Kaźmierczak I. Should patients with obesity be more afraid of COVID-19? Obes Rev. 2020; 21(9):e13083.

30. Popkin BM, Du S, Green WD, Beck MA, Algaith T, Herbst CH, et al. Individuals with obesity and COVID-19: a global perspective on the epidemiology and biological relationships. Obes Rev. 2020;21(11):e13128.

31. Soffer S, Glicksberg BS, Zimlichman E, Efros O, Levin MA, Freeman R, et al. The association between obesity and peak antibody titer response in COVID-19 infection. Obesity. 2021

32. Pasquarelli-do-Nascimento G, Braz-de-Melo HA, Faria SS, IdO S, Kobinger GP, Magalhães KG. Hypercoagulopathy and adipose tissue exacerbated inflammation may explain higher mortality in COVID-19 patients with obesity. Front Endocrinol. 2020;11:530

33. Parameswaran K, Todd DC, Soth M. Altered respiratory physiology in obesity. Can Respir J. 2006:13(4):203-10.

34. O'Rourke RW, Lumeng CN. Pathways to severe COVID-19 for people with obesity. Obesity. 2020

35. Sattar N, Mclnnes IB, McMurray JJ. Obesity is a risk factor for severe COVID19 infection: multiple potential mechanisms. Circulation. 2020;142(1):4-6.

\section{Publisher's Note}

Springer Nature remains neutral with regard to jurisdictional claims in published maps and institutional affiliations.

Ready to submit your research? Choose BMC and benefit from:

- fast, convenient online submission

- thorough peer review by experienced researchers in your field

- rapid publication on acceptance

- support for research data, including large and complex data types

- gold Open Access which fosters wider collaboration and increased citations

- maximum visibility for your research: over $100 \mathrm{M}$ website views per year

At $\mathrm{BMC}$, research is always in progress.

Learn more biomedcentral.com/submissions 